\title{
EHMTI-0184. Ictal adiponectin levels are modulated by pain severity and treatment response in episodic migraineurs
}

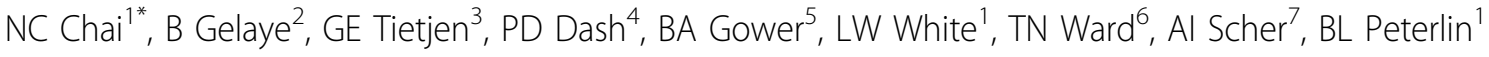 \\ From 4th European Headache and Migraine Trust International Congress: EHMTIC 2014 \\ Copenhagen, Denmark. 18-21 September 2014
}

\section{Introduction}

Adiponection (ADP) and leptin (LEP) are adipokines with roles in inflammation.

\section{Aim}

To assess ADP and LEP levels before and after acute abortive treatment in episodic migraineurs (EM).

\section{Methods}

Peripheral blood specimens were collected from EM participants before and after acute abortive treatment with sumatriptan/naproxen sodium versus placebo.

\section{Results}

A total of 34 participants (17 responders, 17 nonresponders) were included. In all participants, for every 1 point increase in the HMW:T-ADP ratio, pain severity increased by 4.11 (CI: $0.44,7.77 ; \mathrm{p}=0.028$ ). In responders $(\mathrm{n}=17)$, crude T-ADP levels were reduced at $30 \mathrm{~min}$ $(11.49 \pm 3.7 ; \mathrm{p}=0.001), 60 \min (11.54 \pm 3.2 ; \mathrm{p}=0.001)$ and $120 \min (11.39 \pm 3.7 ; \mathrm{p}=0.001)$ after treatment as compared to onset $(12.47 \pm 3.6)$. In non-responders $(\mathrm{n}=$ 17), crude T-ADP levels were unchanged after treatment. After adjustments, T-ADP levels remained decreased 30120 min after treatment in responders; additionally, HMW-ADP, and the HMW:T-ADP ratio were decreased and LMW-ADP and the LMW:T-ADP ratio (coeff 0.04; CI: $0.01,0.07 ; \mathrm{p}=0.043$ ) were increased $120 \mathrm{~min}$ after treatment in responders. In non-responders, the adjusted LMW-ADP (coef -0.45; CI: $-0.77,-0.14 ; \mathrm{p}=0.005$ ) and the LMW:T-ADP ratio (coef -0.04; CI: $-0.07,-0.01 ; \mathrm{p}=0.018$ ) were decreased at $60 \mathrm{~min}$ as well as at $120 \mathrm{~min}$ after treatment. Unadjusted and adjusted LEP levels were not modulated by changes in pain severity or treatment response.

\section{Conclusion}

Adiponectin, but not leptin, is associated with pain severity and is modulated by treatment response in episodic migraineurs.

Conflict of interest.

\begin{abstract}
Authors' details
'Neurology, Johns Hopkins University School of Medicine, Baltimore, USA ${ }^{2}$ Epidemiology, Harvard School of Public Health, Boston, USA. ${ }^{3}$ Neurology, University of Toledo, Toledo, USA. ${ }^{4}$ Neurology, Johns Hopkins Community Physicians, Baltimore, USA. ${ }^{5}$ Department of Nutrition Sciences, University of Alabama at Birmingham, Birmingham, USA. ${ }^{6}$ Neurology, Dartmouth Hitchcock Medical Center, Lebanon, USA. ${ }^{7}$ Preventive Medicine and Biometrics, Uniformed Services University, Bethesda, USA.
\end{abstract}

Published: 18 September 2014

doi:10.1186/1129-2377-15-S1-E25

Cite this article as: Chai et al:: EHMTI-0184. Ictal adiponectin levels are modulated by pain severity and treatment response in episodic migraineurs. The Journal of Headache and Pain 2014 15(Suppl 1):E25. 